\title{
Rapidly Growing Bilateral Pseudoangiomatous Stromal Hyperplasia of the Breast
}

\author{
Eun Mi Ryu, MD' \\ In Yong Whang, $\mathrm{MD}^{1}$ \\ Eun Deok Chang, MD²
}

\section{Index terms: \\ Pseudoangiomatous stromal hyperplasia \\ Breast \\ Sonography \\ Magnetic resonance (MR)}

DOI:10.3348/kjr.2010.11.3.355

Korean J Radiol 2010;11:355-358

Received September 8, 2009; accepted

after revision October 12, 2009.

Departments of 'Radiology and ${ }^{2}$ Clinical Pathology, Uijeongbu St. Mary's Hospital, College of Medicine, The Catholic University of Korea, Gyeonggi-do 480717 , Korea

Address reprint requests to: In Yong Whang, MD, Department of Radiology, Uijeongbu St. Mary's Hospital, College of Medicine, The Catholic University of Korea, 65-1 Geumo-dong, Uijeongbu-si, Gyeonggi-do 480-717, Korea.

Tel. (8231) 820-3749

Fax. (8231) 846-3080

e-mail: tiger@catholic.ac.kr
A tumoral pseudoangiomatous stromal hyperplasia (PASH) that causes huge breast enlargement is very rare. Only two cases of huge tumoral PASHs have been reported in the English medical literature. We report here on a surgically confirmed case of bilateral huge tumoral PASH in a 47-year-old woman, and we present the imaging and histopathology findings. We also review the relevant medical literature. seudoangiomatous stromal hyperplasia is not an uncommon benign lesion of the breast, and the frequency of pseudoangiomatous stromal hyperplasia (PASH) is increasing. The clinical manifestations and imaging findings of PASH are nonspecific. The mammographic findings vary from no identifiable abnormality to a circumscribed mass, and the sonographic findings of most PASHs are a nonspecific, focal, mass-like lesion (1). To the best of our knowledge, a rapidly growing huge tumoral PASH is very rare, although there have been many report of PASH. We report here on a case of bilateral huge tumoral PASH and the patient presented with marked breast enlargement, and we present the imaging and correlative histopathology features.

\section{CASE REPORT}

A 47-year-old woman came to our hospital because of painful swelling of her left breast. She had been suffering from bilateral breast swelling for the previous three months. She also complained of associated bilateral mastalgia for the previous 10 days. On examination, both her breasts showed huge enlargement and the left breast was larger than the right breast. The periareolar skin of both breasts appeared tense, it had undergone erythematous change and the skin was warm to the touch. A discrete mass was not palpated because the abnormality was so large that nearly the entire breast was affected. The axillary lymph nodes were also not palpated. The patient had been diagnosed 15 years previously with diabetes mellitus and she was now blind due to the resulting diabetic retinopathy. The patient was premenopausal and she did not take oral contraceptives or hormonal medications.

Mammography revealed a bilateral, extremely dense, parenchymal pattern. Both breasts were enlarged, although the left breast was larger than the right breast. There was a reticular density surrounding the dense parenchyma and skin thickening without mammographic evidence of a mass. On sonography, skin thickening and low echoes similar to lobular thickening of the glandular layer were noted in both breasts, with the left breast being more prominent, although no discrete mass was detected (Fig. $1 \mathrm{~A}-\mathrm{C})$. The patient required hospital treatment because she was initially diagnosed 
with bilateral diffuse mastitis. After the administration of antibiotics for two days, she was discharged from the hospital as both her breast pain and swelling had subsided.

However, six months later, the patient again complained of the same symptoms. Sonography revealed progression of the previous, lobular echo-like, parenchymal thickening and numerous conglomerations of tiny cysts, although there was no evidence of a mass (Fig. 1D-F). We performed a $14 \mathrm{G}$ core biopsy to confirm the bilateral breast lesions, and PASH was diagnosed (Fig. 1G-I). MRI was performed to evaluate the exact extent of the breast lesion. There were high-signal spaces between the diffuse, nodular low signals on the T2WIs. After contrast
(Gadopentetate dimeglumine) infusion, this diffuse, nodular signal showed homogeneous enhancement. All of the abnormal signals were seen in the entire area of both breasts, although the signals were more prominent in the left breast (Fig. 1J, K, L). The patient subsequently underwent left breast reduction mammoplasty in an attempt to alleviate her symptoms and to correct the breast asymmetry.

\section{DISCUSSION}

Pseudoangiomatous stromal hyperplasia is a benign breast disease that is characterized by a histologically dense proliferation of mammary stroma, which form interanastomosing channels lined by endothelial-like spindle cells.

The exact mechanism of this breast tissue proliferation is unknown, although it is thought to be associated with the

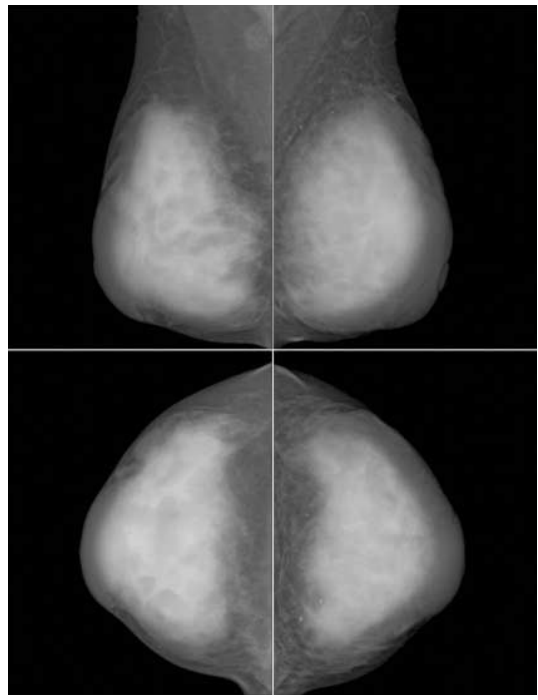

A

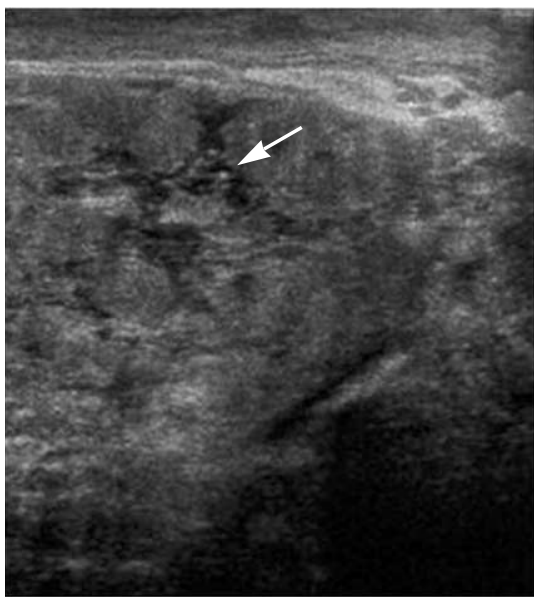

D

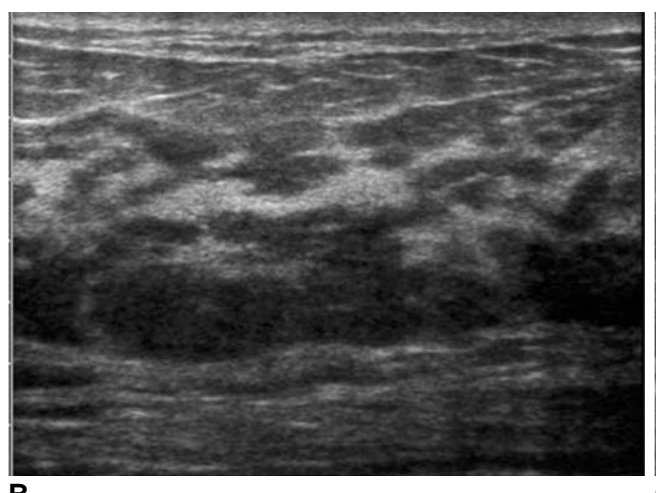

B
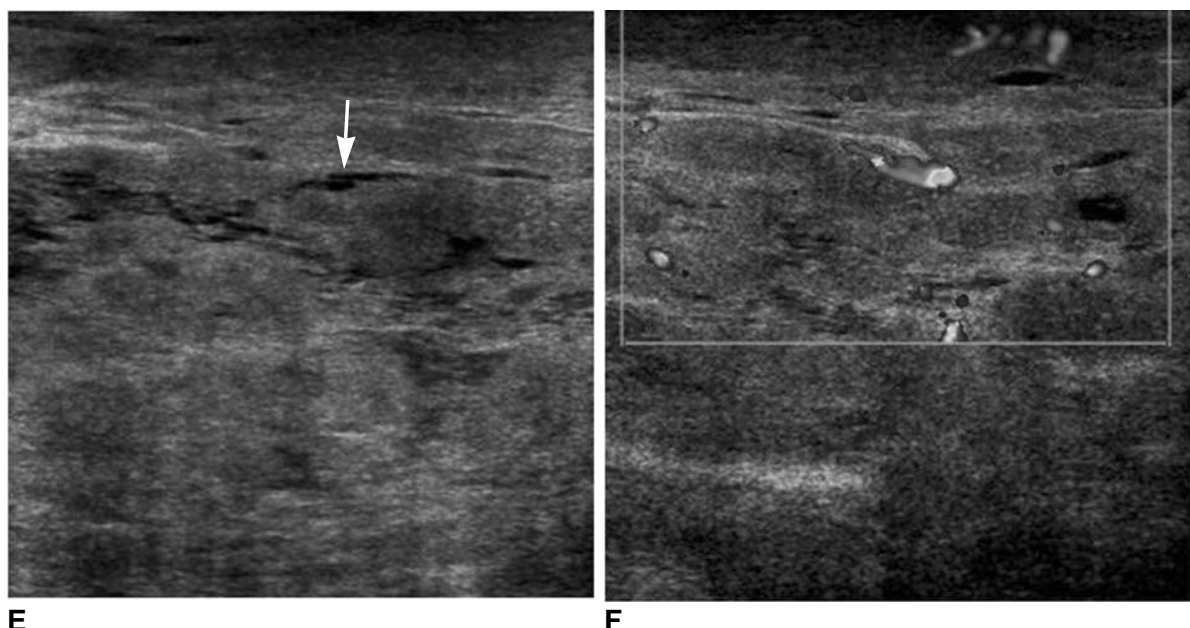

$\mathbf{F}$

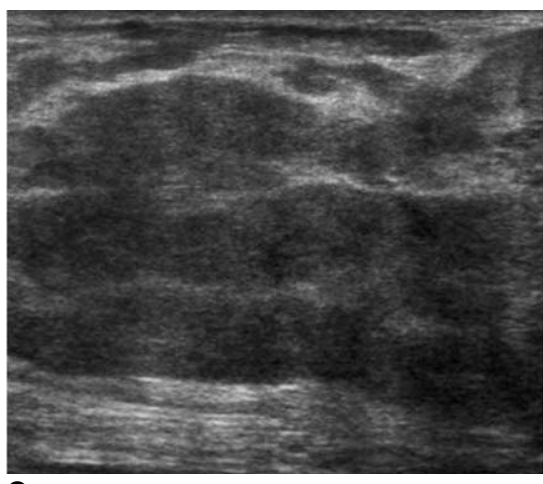

C

Fig. 1. Bilateral pseudoangiomatous stromal hyperplasia of breasts in 47-year-old woman.

A. Initial mammography shows huge enlargement of both breasts. Parenchymal pattern is extremely dense. There is reticular density around parenchyma as well as skin thickening.

B, C. Sonography of right (B) and left $(\mathbf{C})$ breasts shows skin thickening and low echoes similar to those caused by lobular thickening of glandular layer. Left breast is more prominent than right breast.

D-F. Sonography obtained six months after shows substantial progression of glandular layer thickening in patient's left breast. Note presence of conglomerations of tiny cystic spaces (arrows) and abundant color flow indicating hyperemic change. 


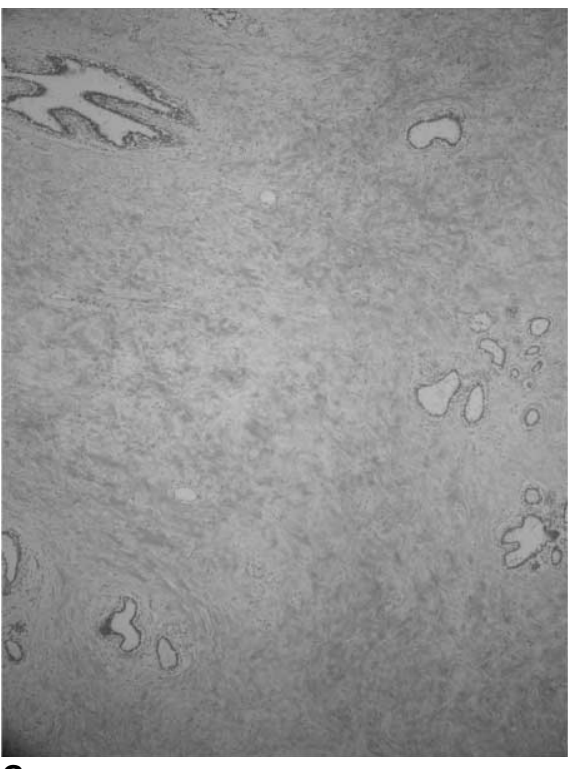

$\mathbf{G}$

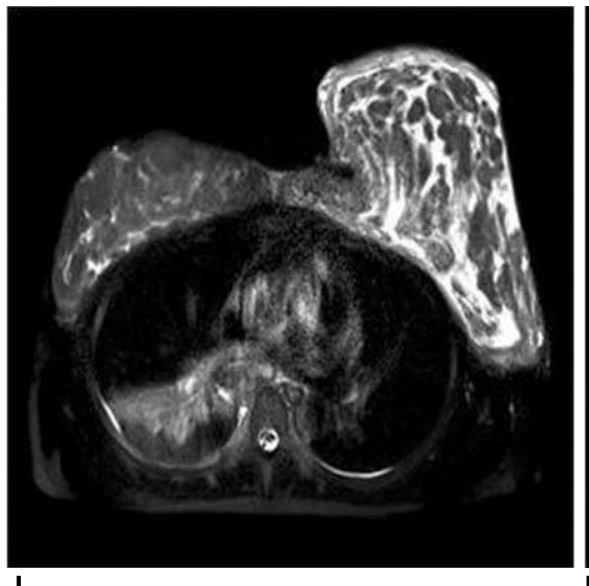

J

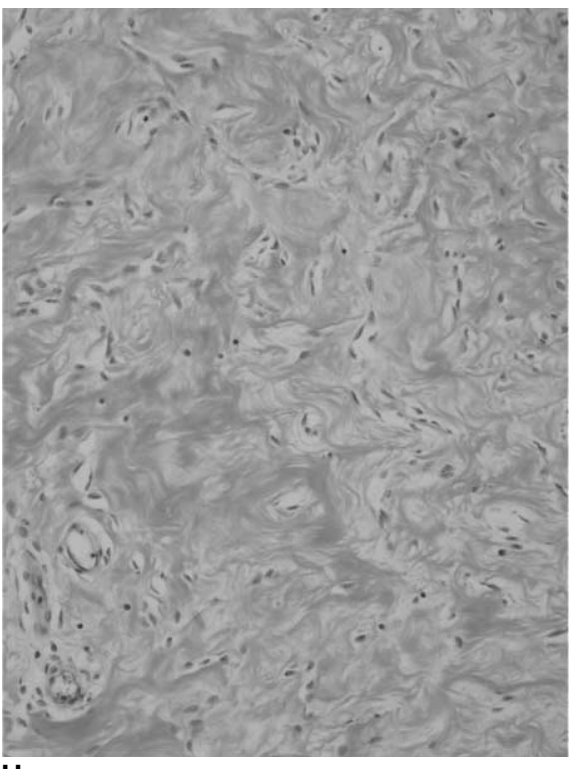

H

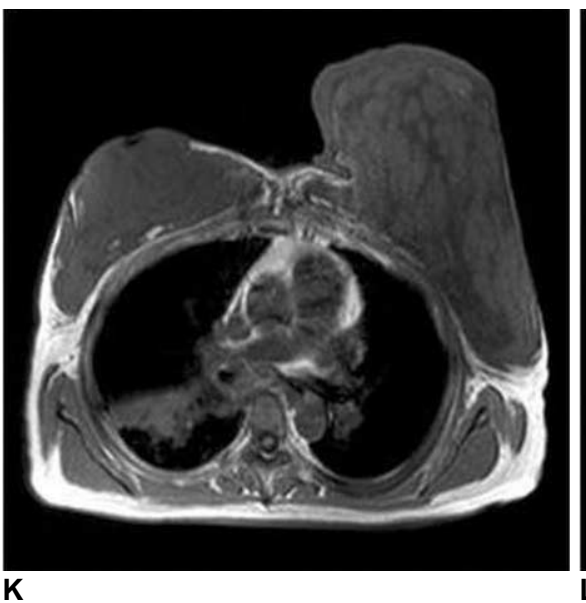

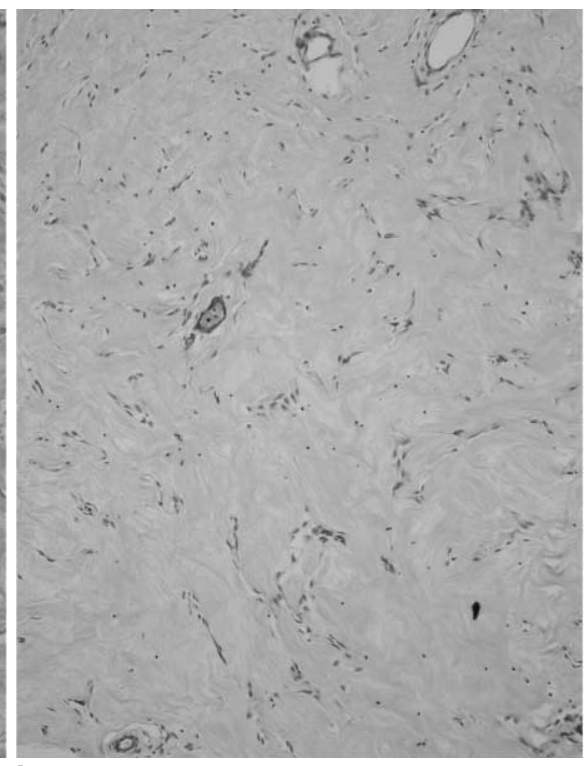

I

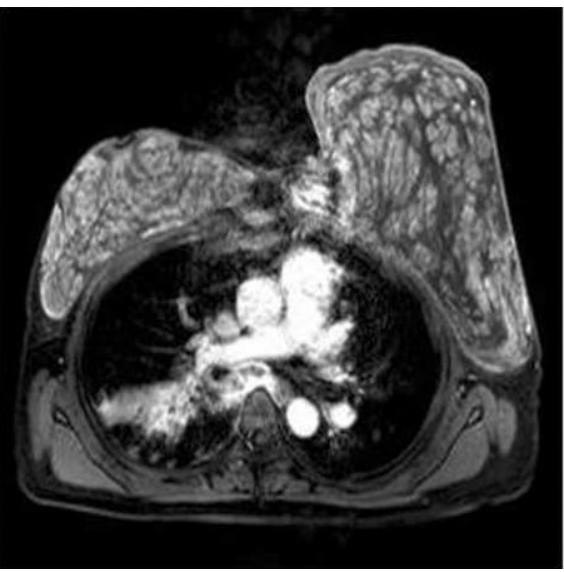

$\mathbf{L}$

Fig. 1. Bilateral pseudoangiomatous stromal hyperplasia of breasts in 47-year-old woman.

G. Classic features of pseudoangiomatous stromal hyperplasia, i.e., dense, fibrous tissue separates lobular architecture with loose, supporting stroma (Hematoxylin \& Eosin staining, $\times 40$ ).

H. Higher magnification view shows cleft lined by endothelial-like, spindle cells in context of stromal hyperplasia (Hematoxylin \& Eosin staining, $\times 200$ ).

I. Immunohistochemistry preparation reveals CD 34 reactivity of pseudovascular space lined by myoepithelial cells (Hematoxylin \& Eosin staining, $\times 100$ ).

J-L. MRI examination of breast shows high-signal spaces between diffuse and nodular low signals on fat-saturated half-fourier acquisition single-shot turbo spin-echo T2 $(\mathbf{J})$. Entire breast reveals low signal on 2D flash T1. Fibrous hyperplasia with cystic spaces is suggested (K). Low signal on T2 or dense, fibrous tissue shows homogeneous enhancement on fat-saturated T1 after contrast infusion (L). Dynamic study using dedicated coil could not be performed.

exaggerated response of the myofibroblasts to hormone stimulation, and especially that of progesterone. This is supported by the fact that PASH usually occurs in young, premenopausal women or in older women who are on estrogen replacement therapy. PASH shows a wide spectrum of findings ranging from relatively frequently seen incidental microfoci, among other pathology findings, to rarely occurring, mass-forming tumors. In one report, PASH was noted in up to one-fourth of the total biopsy specimens (2). However, in those cases where more important pathology findings, such as malignancy, coexist with microfoci of PASH, the description of PASH in the final pathology report may often be omitted.

The term PASH indicates that its histologic appearance is very similar to that of the vascular channels, which are lined by endothelial cells, and the vascular channels contain RBCs (red blood cells). Many reports about PASH have indicated that this factor could also suggest the 
diagnosis of angiosarcoma rather than PASH; however, as pathologists are well aware of both these disease entities, such a misinterpretation should rarely happen.

The subsequent development of carcinoma at the site of PASH does not occur because PASH is a benign lesion and not even a high risk one. However, there is the possibility of coexistent carcinoma, i.e., a small portion of PASH in the vicinity of carcinoma. Therefore, if a suspicious image finding is noted on an image study, further sampling is needed for making the final diagnosis even after PASH is revealed on the core biopsy report. Two, relatively large studies showed that $4 \%$ and $10 \%$ of their total cases, respectively, had coexistent carcinoma at the site of PASH $(1,2)$.

On mammography, PASH can be seen as a non-calcified, circumscribed or partially circumscribed mass or as a spiculated mass or as developing asymmetry when it presents with a solitary or dominantly stromal component. The pathology of PASH can occur in the middle of a surgical scar or radial scar, in ductal carcinoma in situ, in atypical ductal hyperplasia or in fibrocystic change, and any of these could be noted as an architectural distortion or as calcifications on mammography (1, 3-5). In a study by Hargaden et al. (1), PASH was detected on mammography in only $31 \%$ of the 90 patients with palpable PASH lesions, and so this it indicated that some PASH lesions cannot be seen on mammography.

The most frequent sonography finding of tumoral PASH is a well-circumscribed, hypoechoic or isoechoic oval mass with enhanced through transmission; this is very similar to the appearances of benign entities such as fibroadenoma, which are nonspecific $(1,3,4)$. In some cases, an element such as fibrocystic change or adipose tissue could make the lesion more heterogeneous, and the presence of cysts lined by apocrine cells could encourage the formation of small, internal cysts (6).

The MRI appearance of PASH has only been described in extremely rare cases $(7,8)$. According to two reports that used the dedicated breast coil, the stromal tissue or PASH was noted as a low signal and the cystic space in the middle was noted as a high signal on the T2WIs. The
PASH lesions revealed homogeneous enhancement after contrast infusion. The kinetic curve of the persistent enhancement pattern was also been noted in these reports.

In the case of Teh et al. (7), the mammographic presentation of markedly enlarged and extremely dense breasts and the sonography finding of conglomerations of cysts in compact stromal hyperplasia were very similar to our imaging findings.

Although rare, a huge, tumoral PASH should be included in the differential diagnosis of rapidly growing and enlarging breast lesions.

\section{Acknowledgement}

We would like to thank Bonnie Hami, MA (USA) for her editorial assistance in the preparation of the manuscript.

\section{References}

1. Hargaden GC, Yeh ED, Georgian-Smith D, Moore RH, Rafferty EA, Halpern EF, et al. Analysis of the mammographic and sonographic features of pseudoangiomatous stromal hyperplasia. AJR Am J Roentgenol 2008;191:359-363

2. Ibrahim RE, Sciotto CG, Weidner N. Pseudoangiomatous hyperplasia of mammary stroma. Some observations regarding its clinicopathologic spectrum. Cancer 1989;63:1154-1160

3. Polger MR, Denison CM, Lester S, Meyer JE. Pseudoangiomatous stromal hyperplasia: mammographic and sonographic appearances. AJR Am J Roentgenol 1996;166:349-352

4. Cohen MA, Morris EA, Rosen PP, Dershaw DD, Liberman L, Abramson AF. Pseudoangiomatous stromal hyperplasia: mammographic, sonographic, and clinical patterns. Radiology 1996;198:117-120

5. Piccoli CW, Feig SA, Palazzo JP. Developing asymmetric breast tissue. Radiology 1999;211:111-117

6. Mercado CL, Naidrich SA, Hamele-Bena D, Fineberg SA, Buchbinder SS. Pseudoangiomatous stromal hyperplasia of the breast: sonographic features with histopathologic correlation. Breast J 2004;10:427-432

7. Teh HS, Chiang SH, Leung JW, Tan SM, Mancer JF. Rapidly enlarging tumoral pseudoangiomatous stromal hyperplasia in a 15-year-old patient: distinguishing sonographic and magnetic resonance imaging findings and correlation with histologic findings. J Ultrasound Med 2007;26:1101-1106

8. Baskin H, Layfield L, Morrell G. MRI appearance of pseudoangiomatous stromal hyperplasia causing asymmetric breast enlargement. Breast J 2007;13:209-210 